\title{
Rational maps in real algebraic geometry
}

\author{
Wojciech Kucharz* \\ (Communicated by C. Scheiderer)
}

\begin{abstract}
The paper deals with rational maps between real algebraic sets. We are interested in the rational maps which extend to continuous maps defined on the entire source space. In particular, we prove that every continuous map between unit spheres is homotopic to a rational map of such a type. We also establish connections with algebraic cycles and vector bundles.
\end{abstract}

Key words. Rational maps, real algebraic sets, vector bundles.

2000 Mathematics Subject Classification. 14P05, 14P25

\section{Introduction}

Let $X \subseteq \mathbb{R}^{k}$ and $Y \subseteq \mathbb{R}^{\ell}$ be nonsingular irreducible algebraic sets. A map $f: X \longrightarrow Y$ of class $\mathcal{C}^{r}$ (where $r=0,1, \ldots, \infty$, and $\mathcal{C}^{0}$ stands for continuous) is said to be a $\mathcal{C}^{r}$ rational map if there exist a nonempty Zariski open subset $U$ of $X$ and a regular map $\varphi$ : $U \longrightarrow Y$ with $f \mid U=\varphi$. In that case $f$ is completely determined by $\varphi$ since $U$ is dense in $X$. Denote by $P(f)$ the indeterminacy locus (that is, the complement of the domain of definition) of the rational map from $X$ into $Y$ represented by $\varphi$. Hence $P(f)$ is the smallest algebraic subset of $X$ for which the restriction map $f \mid X \backslash P(f): X \backslash P(f) \longrightarrow Y$ is regular. The graph of $f \mid X \backslash P(f)$ is a semi-algebraic subset of $X \times Y$, whose closure is equal to the graph of $f$. It follows that the graph of $f$ is also a semi-algebraic subset. In other words, every $\mathcal{C}^{r}$ rational map is a semi-algebraic map. Thus $\mathcal{C}^{r}$ rational maps are natural objects in real algebraic or semi-algebraic geometry. We shall see below that they have some remarkable properties. We wish to emphasize that certain $\mathcal{C}^{r}$ rational maps admit a very explicit description. Since the restriction $f \mid X \backslash P(f)$ is a regular map, there

\footnotetext{
* The paper was completed at the Max-Planck-Institut für Mathematik in Bonn, whose support and hospitality are gratefully acknowledged.
} 
exist polynomials $P_{1}, \ldots, P_{\ell}, Q$ in $\mathbb{R}\left[T_{1}, \ldots, T_{k}\right]$ with

$$
\begin{aligned}
P(f) & =\left\{x \in \mathbb{R}^{k} \mid Q(x)=0\right\}, \\
f(x) & =\left(\frac{P_{1}(x)}{Q(x)}, \ldots, \frac{P_{\ell}(x)}{Q(x)}\right) \text { for all } x \text { in } X \backslash P(f),
\end{aligned}
$$

cf. [6, Proposition 3.2.3]. Of course, in general, the value of $f$ at a point in $P(f)$ cannot be read off from the formula given above without computing a limit. However, if it happens that $f(P(f))$ contains at most one point, then $f$ is given on the whole $X$ by a finite formula. In several of our results $\mathcal{C}^{r}$ rational maps of exactly such a type are involved.

Let us mention that we obtain a new class of maps exclusively for $r$ finite since any $\mathcal{C}^{\infty}$ rational map is automatically regular, cf. Proposition 2.1 . One readily checks that every continuous rational map from $X$ into $Y$ is regular, provided $\operatorname{dim} X=1$. Some explicit examples of $\mathcal{C}^{r}$ rational maps that are not regular are given in Example 2.2. In this paper we deal with problems specific to real algebraic geometry. Any continuous rational map between complex algebraic sets is regular.

Henceforth we mainly investigate under what conditions a continuous map from $X$ into $Y$ is homotopic to a $\mathcal{C}^{r}$ rational map. For maps between unit spheres the answer is definitive: no extra assumptions are necessary. More precisely, let $S^{n}$ denote the unit $n$-sphere,

$$
S^{n}=\left\{\left(x_{1}, \ldots, x_{n+1}\right) \in \mathbb{R}^{n+1} \mid x_{1}^{2}+\cdots+x_{n+1}^{2}=1\right\} .
$$

Theorem 1.1. Let $r$ be a nonnegative integer. For every pair $(n, p)$ of positive integers, each continuous map $h: S^{n} \rightarrow S^{p}$ is homotopic to a $\mathcal{C}^{r}$ rational map $f: S^{n} \rightarrow S^{p}$ with $\operatorname{codim}_{S^{n}} P(f) \geq p$ and $f(P(f))$ containing at most one point.

In the literature there are some related results concerning polynomial or regular maps between spheres. The motivation was to represent homotopy classes of maps between spheres by "simple" maps, given by finite formulas. As it turned out, this cannot always be achieved with polynomial maps. Making use of quadratic forms, Wood [25] proved that every polynomial map from $S^{n}$ into $S^{p}$ is a constant map, provided $n \geq 2^{m}>p$ for some integer $m$. The question whether every continuous map from $S^{n}$ into $S^{p}$ is homotopic to a regular map, for all pairs $(n, p)$, remains open and seems to be a hard problem, cf. [6, p. 367] and [22, p. 1153]. According to [6, 7, 8, 22], the answer is affirmative for some pairs $(n, p)$ with the homotopy group $\pi_{n}\left(S^{p}\right)$ nontrivial, including infinitely many such pairs for which the polynomial maps are constant.

Theorem 1.1 is proved in Section 2, which contains also results concerning $\mathcal{C}^{r}$ rational maps from $X$ into $S^{p}$. Among the latter the following is the simplest.

Theorem 1.2. Let $r$ be a nonnegative integer. Assume that $X$ is compact and $\operatorname{dim} X=n$. Then each continuous map $h: X \rightarrow S^{n}$ is homotopic to a $\mathcal{C}^{r}$ rational map $f: X \rightarrow S^{n}$ with the set $P(f)$ finite and $f(P(f))$ containing at most one point.

This result demonstrates that $\mathcal{C}^{r}$ rational maps are indeed more flexible than regular maps. For example, in contrast with Theorem 1.2, a continuous map from $S^{1} \times S^{1}$ into $S^{2}$ is homotopic to a regular map if and only if it is null homotopic, cf. [6, 8]. Some 
cases, resolved by Theorem 1.2 for $\mathcal{C}^{r}$ rational maps, remain open problems for regular maps. For instance, it is not known whether every continuous map from $S^{2} \times S^{2}$ into $S^{4}$ is homotopic to a regular map, cf. [6, Remark 12.5.5].

In general not every continuous map from $X$ into $S^{p}$ is homotopic to a $\mathcal{C}^{r}$ rational map. Some obstructions depend on the existence of homology or cohomology classes not representable by algebraic subsets. Below we make this precise.

Assume that $X$ is compact. Denote by

$$
H_{d}^{\mathrm{alg}}(X, \mathbb{Z} / 2)
$$

the subgroup of the homology group $H_{d}(X, \mathbb{Z} / 2)$ generated by the homology classes represented by $d$-dimensional algebraic subsets of $X$, cf. $[4,6,13,14]$. As usual, set

$$
H_{\text {alg }}^{c}(X, \mathbb{Z} / 2)=D_{X}^{-1}\left(H_{d}^{\text {alg }}(X, \mathbb{Z} / 2)\right),
$$

where $c+d=\operatorname{dim} X$ and $D_{X}: H^{c}(X, \mathbb{Z} / 2) \rightarrow H_{d}(X, \mathbb{Z} / 2)$ is the Poincaré duality isomorphism. Given any continuous map $h: X \rightarrow Y$, denote by

$$
h_{*}: H_{d}(X, \mathbb{Z} / 2) \rightarrow H_{d}(Y, \mathbb{Z} / 2) \quad \text { and } \quad h^{*}: H^{c}(Y, \mathbb{Z} / 2) \rightarrow H^{c}(X, \mathbb{Z} / 2)
$$

the induced homomorphisms.

Proposition 1.3. Assume that $X$ and $Y$ are compact. If $f: X \rightarrow Y$ is a continuous rational map, then

$$
\begin{gathered}
f_{*}\left(H_{d}^{\mathrm{alg}}(X, \mathbb{Z} / 2)\right) \subseteq H_{d}^{\mathrm{alg}}(Y, \mathbb{Z} / 2), \\
f^{*}\left(H_{\mathrm{alg}}^{c}(Y, \mathbb{Z} / 2)\right) \subseteq H_{\mathrm{alg}}^{c}(X, \mathbb{Z} / 2)
\end{gathered}
$$

for $d \geq 0$ and $c \geq 0$.

Proposition 1.3 is well known for regular maps (cf. [4, 6, 13, 14]), and therefore it holds for continuous maps homotopic to regular ones. There are however $\mathcal{C}^{r}$ rational maps not homotopic to regular maps, cf. Theorem 1.2 and the comment that follows it.

Denote by $\sigma_{p}$ the unique generator of $H_{\text {alg }}^{p}\left(S^{p}, \mathbb{Z} / 2\right)=H^{p}\left(S^{p}, \mathbb{Z} / 2\right) \cong \mathbb{Z} / 2$. By Proposition 1.3, a necessary condition for a continuous map $h: X \rightarrow S^{p}$ to be homotopic to a continuous rational map is that $h^{*}\left(\sigma_{p}\right)$ be in $H_{\mathrm{alg}}^{p}(X, \mathbb{Z} / 2)$. In some cases this condition is also sufficient.

Corollary 1.4. Assume that $X$ is compact. For any continuous map $h: X \rightarrow S^{1}$, the following conditions are equivalent:

(a) $h$ is homotopic to a continuous rational map.

(b) $h$ is homotopic to a regular map.

(c) $h$ can be approximated by regular maps.

(d) $h^{*}\left(\sigma_{1}\right)$ is in $H_{\text {alg }}^{1}(X, \mathbb{Z} / 2)$.

Proof. It is known that Conditions (b), (c), (d) are equivalent, cf. [7, Theorem 1.4]. By Proposition 1.3, (a) implies (d), while (b) trivially implies (a). 
Regular maps with values in $S^{1}$ are extensively studied in [6, 10]. It is known that Condition (d) is an essential restriction.

Theorem 1.5. Let $r$ be a nonnegative integer. Assume that $X$ is compact and $\operatorname{dim} X=$ $n+1$. For any continuous map $h: X \rightarrow S^{n}$, the following conditions are equivalent:

(a) $h$ is homotopic to a $\mathcal{C}^{r}$ rational map $f: X \rightarrow S^{n}$ with $\operatorname{dim} P(f) \leq 1$ and $f(P(f))$ containing at most one point.

(b) $h$ is homotopic to a continuous rational map.

(c) $h^{*}\left(\sigma_{n}\right)$ is in $H_{\mathrm{alg}}^{n}(X, \mathbb{Z} / 2)$.

Other results concerning representation of homotopy classes of maps from $X$ into $S^{p}$ by $\mathcal{C}^{r}$ rational maps are contained in Sections 2 and 3, cf. Theorems 2.4, 2.5, Proposition 2.8, and Corollaries 2.6, 2.7, 3.8. Among these Theorem 2.4 is crucial. All results announced above are proved in Section 2. In Section 3 we study $\mathcal{C}^{r}$ rational maps with values in Grassmannians. To this end we introduce the notion, interesting in its own right, of $\mathcal{C}^{r}$ rational structure on a vector bundle.

In order to allow for ease of exposition it will be convenient to adopt in the subsequent sections the following convention. The term real algebraic variety will designate a locally ringed space isomorphic to an algebraic subset of $\mathbb{R}^{n}$, for some $n$, endowed with the Zariski topology and the sheaf of $\mathbb{R}$-valued regular functions. Recall that the quasi-projective real algebraic varieties are real algebraic varieties in this sense, cf. [6, Proposition 3.2.10, Theorem 3.4.4]. Zariski closed subsets of a real algebraic variety will be often called algebraic subsets. Morphisms between real algebraic varieties will be called regular maps. Every real algebraic variety carries also the Euclidean topology, which is determined by the usual metric topology on $\mathbb{R}$. Unless explicitly stated otherwise, all topological notions related to real algebraic varieties will refer to the Euclidean topology. Our standard reference on real algebraic geometry is [6].

\section{Rational maps into spheres}

We begin by showing that $\mathcal{C}^{\infty}$ rational maps are regular maps. The argument is completely standard.

Proposition 2.1. Let $X$ and $Y$ be nonsingular irreducible real algebraic varieties. Any $\mathcal{C}^{\infty}$ rational map from $X$ into $Y$ is a regular map.

Proof. We may assume $Y \subseteq \mathbb{R}^{\ell}$, for some $\ell$, and hence reduce the proof to the case $Y=\mathbb{R}$. Let $f: X \rightarrow \mathbb{R}$ be a $\mathcal{C}^{\infty}$ rational function. There exist two regular functions $\varphi$ and $\psi$ from $X$ into $\mathbb{R}$ with $\psi^{-1}(0) \neq X$ and $f(x)=\varphi(x) / \psi(x)$ for all $x$ in $X \backslash \psi^{-1}(0)$. Thus $\psi f=\varphi$ on $X$.

Given a point $x$ in $X$, we denote by $\mathcal{R}_{x}$ the local ring of $X$ at $x$, that is, the ring of germs at $x$ (with respect to the Zariski topology) of regular functions. Since $X$ is nonsingular and irreducible, the completion $\hat{\mathcal{R}}_{x}$ of $\mathcal{R}_{x}$, with respect to the maximal ideal of $\mathcal{R}_{x}$, is isomorphic to the ring of formal power series in $n$ variables over $\mathbb{R}, n=\operatorname{dim} X$. 
The equality $\psi f=\varphi$ implies that $\psi_{x}$ divides $\varphi_{x}$ in $\hat{\mathcal{R}}_{x}$, where $\varphi_{x}$ and $\psi_{x}$ are the germs at $x$ of, respectively, $\varphi$ and $\psi$. Hence $\psi_{x}$ divides $\varphi_{x}$ in $\mathcal{R}_{x}$, cf. [21, 1 1.C]. Consequently, $f$ is a regular function in a Zariski neighborhood of $x$. Since $x$ is an arbitrary point of $X$, it follows that $f$ is regular on $X$.

In view of Proposition 2.1, considering $\mathcal{C}^{r}$ rational maps we will usually assume that $r$ is finite.

Example 2.2. Assume that $r$ is a nonnegative integer.

(i) There exists a $\mathcal{C}^{r}$ rational map $g: S^{2} \rightarrow S^{1}$ with $P(g) \neq \emptyset$. Indeed, $h: \mathbb{R}^{2} \rightarrow \mathbb{R}$,

$$
h\left(x_{1}, x_{2}\right)= \begin{cases}\frac{\left(x_{1}^{4}+x_{2}^{4}\right)^{r+1}}{x_{1}^{2}+x_{2}^{2}} & \text { for }\left(x_{1}, x_{2}\right) \neq(0,0) \\ 0 & \text { for }\left(x_{1}, x_{2}\right)=(0,0)\end{cases}
$$

is a $\mathcal{C}^{r}$ rational function with $P(h)=\{(0,0)\}$. Define $g: S^{2} \rightarrow S^{1}$ by $g=\sigma \circ h \circ \pi$, where

$$
\begin{aligned}
& \pi: S^{2} \rightarrow \mathbb{R}^{2}, \pi\left(x_{1}, x_{2}, x_{3}\right)=\left(x_{1}, x_{2}\right), \\
& \sigma: \mathbb{R} \rightarrow S^{1}, \sigma(t)=\left(\frac{2 t}{t^{2}+1}, \frac{t^{2}-1}{t^{2}+1}\right) .
\end{aligned}
$$

Then $g$ is a $\mathcal{C}^{r}$ rational map with $P(g)=\{(0,0,1),(0,0,-1)\}$.

(ii) There exists a $\mathcal{C}^{r}$ rational map $f: S^{2} \times S^{1} \rightarrow S^{1}$ such that $f$ is not null homotopic and $f(P(f))=S^{1}$. Indeed, define $f$ by $f(x, z)=g(x) z$ for $(x, z)$ in $S^{2} \times S^{1}$, where $g$ is as in (i) and $S^{1}=\{z \in \mathbb{C}|| z \mid=1\}$ is regarded as a multiplicative group. It follows that $P(f)=P(g) \times S^{1}$, and hence $f(P(f))=S^{1}$. Moreover, $f$ is not null homotopic since for any point $x$ in $S^{2}$, the map $S^{1} \rightarrow S^{1}, z \rightarrow f(x, z)$ is homotopic to the identity map.

As the first step toward our main goals, we record a simple fact concerning nonsingular points of real algebraic varieties.

Lemma 2.3. Let $X$ be a nonsingular real algebraic variety and let $U$ be a nonempty Zariski open subset of $X$. Let $N$ be a Zariski closed subset of $U$ and let $V$ be its Zariski closure in $X$. Then $V=N \cup W$, where $W$ is a Zariski closed subset of $X$ with $N \cap W=\emptyset$ and $\operatorname{dim} W<\operatorname{dim} N$. In particular, $N$ is precisely the set of nonsingular points of $V$, assuming $N$ is compact and nonsingular.

Proof. The equality $\operatorname{dim} N=\operatorname{dim} V$ is well known. Since $N=V \cap U$, the set $W=$ $V \backslash N$ is Zariski closed in $X$ and $W \subseteq X \backslash U$. We have $V=N \cup W, N \cap W=\emptyset$, and $\operatorname{dim} W \leq \operatorname{dim} V$. Moreover, no irreducible component of $V$ is contained in $W$. It follows that $\operatorname{dim} W<\operatorname{dim} V$, as required. Indeed, suppose to the contrary that $\operatorname{dim} W=\operatorname{dim} V$ and choose an irreducible component $Z$ of $W$ with $\operatorname{dim} Z=\operatorname{dim} V$. Then $Z$ is an irreducible component of $V$, and we get a contradiction.

The last assertion in the lemma follows immediately. 
Proofs of the results announced in Section 1 depend on the Pontryagin-Thom construction. Unless explicitly specified otherwise, all smooth (of class $\mathcal{C}^{\infty}$ ) manifolds will be without boundary. Smooth submanifolds will be closed subsets of the ambient manifold. The unit $p$-sphere $S^{p}$ will be oriented as the boundary of the unit $(p+1)$-disk. Recall that for any compact smooth manifold $M$ there is a canonical one-to-one correspondence

$$
\pi^{p}(M) \longrightarrow F^{p}(M)
$$

where $\pi^{p}(M)$ is the set of homotopy classes of continuous maps from $M$ into $S^{p}$, and $F^{p}(M)$ is the set of framed cobordism classes of framed submanifolds of $M$ of codimension $p$ (cf. $[15,19]$ for details). Given a continuous map $h: M \rightarrow S^{p}$, we denote by

$$
P T(h)
$$

the element of $F^{p}(M)$ corresponding to the homotopy class of $h$.

For sake of clarity, it will be convenient to introduce some notation related to this construction. A framed submanifold of $M$ of codimension $p$ is a pair $(N, F)$, where $N$ is a codimension $p$ smooth submanifold of $M$ with trivial normal bundle $\nu(N)$, while $F=\left(v_{1}, \ldots, v_{p}\right)$ is a $p$-tuple of smooth sections of $\nu(N)$ such that $\left(v_{1}(x), \ldots, v_{p}(x)\right)$ is a basis of the fiber $\nu(N)_{x}$ of $\nu(N)$ over $x$ for every point $x$ in $N$. Here $\nu(N)$ is regarded as the quotient vector bundle $(\tau(M) \mid N) / \tau(N)$, where $\tau(M)$ (respectively $\tau(N)$ ) is the tangent bundle to $M$ (respectively $N$ ).

Given a continuous map $h: M \rightarrow S^{p}$ and a point $y$ in $S^{p}$, assume the existence of an open neighborhood $V$ of $y$ for which the restriction map $h \mid h^{-1}(V): h^{-1}(V) \rightarrow V$ is smooth and transverse to $y$. Choose a positively oriented basis $B=\left(w_{1}, \ldots, w_{p}\right)$ of the tangent space $\tau\left(S^{p}\right)_{y}$. Then $P T(h)$ is represented by the framed submanifold $\left(h^{-1}(y), F(h, B)\right)$, where $F(h, B)=\left(v_{1}, \ldots, v_{p}\right)$ and $\left(v_{1}(x), \ldots, v_{p}(x)\right)$ is transformed onto $\left(w_{1}, \ldots, w_{p}\right)$ by the isomorphism $\nu(N)_{x} \rightarrow \tau\left(S^{p}\right)_{y}$ induced by the derivative $d h_{x}$ : $\tau(M)_{x} \rightarrow \tau\left(S^{p}\right)_{y}$ for every $x$ in $h^{-1}(y)$.

Let $\varphi: M \rightarrow \mathbb{R}^{p}$ be a smooth map transverse to 0 in $\mathbb{R}^{p}$ and let $N$ be the union of some connected components of $\varphi^{-1}(0)$. Then we obtain a framed submanifold $(N, F(\varphi))$ of $M$, where $F(\varphi)=\left(v_{1}, \ldots, v_{p}\right)$ and $\left(v_{1}(x), \ldots, v_{p}(x)\right)$ is transformed onto the canonical basis of $\mathbb{R}^{p}$ by the isomorphism $\nu(N)_{x} \rightarrow \mathbb{R}^{p}$ induced by the derivative $d \varphi_{x}$ : $\tau(M)_{x} \rightarrow \tau\left(\mathbb{R}^{p}\right)_{0}=\mathbb{R}^{p}$ for every $x$ in $N$.

The following is the main result of this section.

Theorem 2.4. Let $r$ be a nonnegative integer. For any compact nonsingular irreducible real algebraic variety $X$ and any continuous map $h: X \rightarrow S^{p}$, the following conditions are equivalent:

(a) $h$ is homotopic to a $\mathcal{C}^{r}$ rational map $f: X \rightarrow S^{p}$ with $\operatorname{codim}_{X} P(f) \geq p$ and $f(P(f))$ containing at most one point.

(b) $h$ is homotopic to a continuous rational map $g: X \rightarrow S^{p}$ with $g(P(g)) \neq S^{p}$.

(c) $P T(h)$ is represented by a framed submanifold $(N, F)$ of $X$, where $N$ is the set of nonsingular points of some algebraic subset of $X$. 
Proof. Obviously, (a) implies (b). Suppose that (b) holds. Since $g(P(g))$ is a proper closed subset of $S^{p}$, it follows from Sard's Theorem that the regular map $g \mid X \backslash P(g)$ : $X \backslash P(g) \rightarrow S^{p}$ is transverse to some point $y$ in $S^{p} \backslash g(P(g))$. Hence $P T(h)=P T(g)$ is represented by the framed submanifold $\left(g^{-1}(y), F(g, B)\right)$, where $B$ is a positively oriented basis of $\tau\left(S^{p}\right)_{y}$. Note that $g^{-1}(y)$ is a nonsingular Zariski closed subset of $X \backslash P(g)$. By Lemma 2.3, $g^{-1}(y)$ is precisely the set of nonsingular points of the Zariski closure of $g^{-1}(y)$ in $X$. Consequently, (c) is satisfied with $(N, F)=\left(g^{-1}(y), F(g, B)\right)$.

It remains to prove that (c) implies (a). This follows immediately from Theorem 2.5 below, which contains also some additional information.

We introduce first some terminology. Let $X$ be a nonsingular real algebraic variety and let $S$ be an algebraic subset of $X$. Suppose there is a finite sequence of maps

$$
Y=X_{k} \stackrel{\pi_{k}}{\longrightarrow} X_{k-1} \stackrel{\pi_{k-1}}{\longrightarrow} \cdots \stackrel{\pi_{2}}{\longrightarrow} X_{1} \stackrel{\pi_{1}}{\longrightarrow} X_{0}=X,
$$

where $\pi_{1}$ is the blowup of $X_{0}$ at a nonsingular algebraic subset of $X_{0}$ contained in $S$ and $\pi_{i+1}$ is the blowup of $X_{i}$ at a nonsingular algebraic subset of $X_{i}$ contained in $\left(\pi_{1} \circ \cdots \circ\right.$ $\left.\pi_{i}\right)^{-1}(S)$ for $i=1, \ldots, k-1$. We call the composite map $\pi=\pi_{1} \circ \cdots \circ \pi_{k}: Y \rightarrow X$ a multiblowup of $X$ over $S$. Note that $Y$ is a nonsingular real algebraic variety and the restriction $\pi_{S}: Y \backslash \pi^{-1}(S) \rightarrow X \backslash S$ of $\pi$ is a biregular isomorphism. In particular, if $A$ is an algebraic subset of $Y$, then $\pi(A) \cup S$ is an algebraic subset of $X$.

Theorem 2.5. Let $X$ be a compact nonsingular irreducible real algebraic variety and let $(N, F)$ be a framed submanifold of $X$ of codimension $p$. Assume that $N$ is the set of nonsingular points of some algebraic subset of $X$. Then for any nonnegative integer $r$, there exists a $\mathcal{C}^{r}$ rational map $f: X \rightarrow S^{p}$ such that

(i) $\operatorname{codim}_{X} P(f) \geq p$,

(ii) $f(P(f)) \subseteq\{a\}$,

(iii) $f^{-1}(b)=N$,

(iv) $f \mid X \backslash P(f): X \backslash P(f) \rightarrow S^{p}$ is transverse to $b$,

(v) $(N, F(f, B))$ is framed cobordant to $(N, F)$,

where $a=(0, \ldots, 0,1) \in S^{p}, b=(0, \ldots, 0,-1) \in S^{p}$ and $B$ is a positively oriented basis of the tangent space $\tau\left(S^{p}\right)_{b}$.

Proof. Let $Z$ be the Zariski closure of $N$ in $X$. By assumption, $N$ is the set of nonsingular points of $Z$. In particular, $S:=Z \backslash N$ is an algebraic subset of $X$. The singularities of $Z$ can be resolved by a finite sequence of blowups. More precisely, by Hironaka's resolution of singularities theorem [16], there exists a multiblowup $\pi: Y \rightarrow X$ of $X$ over $S$ such that the Zariski closure $V$ of $\pi^{-1}(N)$ in $Y$ is nonsingular. We assert $V=\pi^{-1}(N)$. Indeed, $Y \backslash \pi^{-1}(S)$ is a Zariski open subset of $Y$ and $\pi^{-1}(N)$ is a Zariski closed subset of $Y \backslash \pi^{-1}(S)$. Consequently, $\operatorname{dim}\left(V \backslash \pi^{-1}(N)\right)<\operatorname{dim} \pi^{-1}(N)$ (cf. Lemma 2.3). Hence $\pi^{-1}(N)$ is dense in $V$ in the Euclidean topology, $V$ being nonsingular. The set $N$ is compact, so the last observation implies $\pi(V) \subseteq N$, and therefore $V=\pi^{-1}(N)$, as asserted. 
In particular, $Y$ is a compact nonsingular irreducible real algebraic variety and $V$ is a nonsingular algebraic subset of $Y$. Since the restriction

$$
\pi_{S}: Y \backslash \pi^{-1}(S) \rightarrow X \backslash S
$$

of $\pi$ is a biregular isomorphism, the normal bundle of $V$ in $Y$ is trivial. We endow $V$ with a framing $F_{V}$ so that the framed submanifold $\left(V, F_{V}\right)$ of $Y$ corresponds to $(N, F)$ via $\pi_{S}$. A standard transversality argument implies the existence of a smooth map $\varphi: Y \rightarrow \mathbb{R}^{p}$ such that $\varphi$ is transverse to 0 in $\mathbb{R}^{p}, V \subseteq \varphi^{-1}(0)$ (in particular, $V$ is the union of some connected components of $\left.\varphi^{-1}(0)\right)$, and $(V, F(\varphi))$ is framed cobordant to $\left(V, F_{V}\right)$. By a relative version of the Weierstrass approximation theorem [6, Lemma 12.5.5], one can find a regular map $\psi: Y \rightarrow \mathbb{R}^{p}$ arbitrarily close in the $\mathcal{C}^{\infty}$ topology to $\varphi$ and with $V \subseteq \psi^{-1}(0)$. If $\psi$ is sufficiently close to $\varphi$, then $\psi$ is transverse to 0 in $\mathbb{R}^{p}$ and $(V, F(\psi))$ is framed cobordant to $(V, F(\varphi))$. Moreover, $\psi^{-1}(0)$ is a nonsingular algebraic subset of $Y$ and

$$
\psi^{-1}(0)=V \cup W
$$

where $W$ is a subset of $Y$ with $V \cap W=\emptyset$. Since $V$ is also a nonsingular algebraic subset of $Y$, it follows that $W$ is an algebraic subset of $Y$, cf. [6, Proposition 3.3.17]. Hence $W \cup \pi^{-1}(S)$ is an algebraic subset of $Y$, which implies the existence of a regular function $\alpha: Y \rightarrow \mathbb{R}$ with

$$
\alpha^{-1}(0)=W \cup \pi^{-1}(S) .
$$

Note that $V \cap \alpha^{-1}(0)=\emptyset$. By the Łojasiewicz inequality [6, Corollary 2.6.7], there exist a neighborhood $U$ of $\alpha^{-1}(0)$ in $Y$, a positive real number $c$, and a positive integer $k$ satisfying

$$
\|\psi(y)\| \geq c \alpha(y)^{2 k} \text { for all } y \text { in } U,
$$

where \|\| denotes the Euclidean norm on $\mathbb{R}^{p}$. Set

$$
\beta(y)=1 / \alpha(y)^{2(k+\ell)} \text { for all } y \text { in } Y \backslash \alpha^{-1}(0),
$$

where $\ell$ is a positive integer to be determined later.

The stereographic projection

$$
\rho: S^{p} \backslash\{a\} \rightarrow \mathbb{R}^{p}
$$

is a biregular isomorphism. Define $h: Y \rightarrow S^{p}$ by

$$
h(y)= \begin{cases}\rho^{-1}(\beta(y) \psi(y)) & \text { for } y \text { in } Y \backslash \alpha^{-1}(0) \\ a & \text { for } y \text { in } \alpha^{-1}(0) .\end{cases}
$$

By construction, the restriction $h \mid Y \backslash \alpha^{-1}(0)$ is a regular map transverse to $b$. Moreover, $h$ is continuous, $h^{-1}(b)=V$, and $(V, F(h, B))$ is framed cobordant to $(V, F(\psi))$. Thus $(V, F(h, B))$ is framed cobordant to $\left(V, F_{V}\right)$.

Note that $\pi\left(\alpha^{-1}(0)\right)=\pi(W) \cup S$ is an algebraic subset of $X$ with $\operatorname{codim}_{X} \pi\left(\alpha^{-1}(0)\right)$ $\geq p$. Define $f: X \rightarrow S^{p}$ by

$$
f(x)= \begin{cases}\left(h \circ \pi_{S}^{-1}\right)(x) & \text { for } x \text { in } X \backslash \pi\left(\alpha^{-1}(0)\right) \\ a & \text { for } x \text { in } \pi\left(\alpha^{-1}(0)\right) .\end{cases}
$$


Then $f \mid X \backslash \pi\left(\alpha^{-1}(0)\right)$ is a regular map transverse to $b$. Since $Y$ is compact and $\pi$ : $Y \rightarrow X$ is a continuous map, it follows that $f$ is also continuous. By construction, $f^{-1}(b)=N$ and $(N, F(f, B))$ is framed cobordant to $(N, F)$. The last assertion holds since $(V, F(h, B))$ is framed cobordant to $\left(V, F_{V}\right)$, and $\left(V, F_{V}\right)$ corresponds to $(N, F)$ via $\pi_{S}$. Clearly, $P(f) \subseteq \pi\left(\alpha^{-1}(0)\right)$, and hence $\operatorname{codim}_{X} P(f) \geq p$ and $f(P(f)) \subseteq\{a\}$. It remains to observe that $f$ is of class $\mathcal{C}^{r}$ if $\ell$ is a sufficiently large integer. This follows from the representation of blowups in local coordinates.

Proof of Theorem 1.1. Let $\left(M, F_{M}\right)$ be a framed submanifold of $S^{n}$ representing $P T(h)$. We may assume that the point $a=(0, \ldots, 0,1) \in S^{n}$ is not in $M$, and hence $\rho(M)$ is a smooth submanifold of $\mathbb{R}^{n}$, where $\rho: S^{n} \backslash\{a\} \rightarrow \mathbb{R}^{n}$ is the stereographic projection. By [2, Theorem A], $\rho(M)$ is isotopic in $\mathbb{R}^{n}$ to the set of nonsingular points of some algebraic subset $W$ of $\mathbb{R}^{n}$. Denoting by $V$ the Zariski closure of $\rho^{-1}(W)$ in $S^{n}$, we have $V \subseteq \rho^{-1}(W) \cup\{a\}$. Thus $M$ is isotopic in $S^{n}$ to the set $N$ of nonsingular points of $V$. Consequently, there is a framing $F$ of $N$ such that $(N, F)$ is framed cobordant to $\left(M, F_{M}\right)$. Since $(N, F)$ represents $P T(h)$, the proof is complete in view of Theorem 2.4.

Proof of Theorem 1.2. It suffices to apply Theorem 2.4.

The other results stated in Section 1 could be proved now, but instead we derive first three consequences of Theorem 2.4. These facts are not required for the proofs of Proposition 1.3 and Theorem 1.5.

Corollary 2.6. Let $X$ be a compact nonsingular irreducible real algebraic variety and let $p$ be a positive integer. Assume that $X$ is homotopically equivalent to $S^{n}, n \geq 1$. Then for any nonnegative integer $r$, each continuous map $h: X \rightarrow S^{p}$ is homotopic to a $\mathcal{C}^{r}$ rational map $f: X \rightarrow S^{p}$ with $\operatorname{codim}_{X} P(f) \geq p$ and $f(P(f))$ containing at most one point.

Proof. Let $\varphi: X \rightarrow S^{n}$ be a homotopy equivalence and let $g: S^{n} \rightarrow S^{p}$ be a continuous map such that $h$ is homotopic to $g \circ \varphi$. By Theorem 1.1, we may assume that $g$ is a $\mathcal{C}^{r}$ rational map with $g(P(g))$ containing at most one point. Similarly, by Theorem 2.4, we may assume that $\varphi$ is a $\mathcal{C}^{r}$ rational map with $\varphi(P(\varphi))$ containing at most one point. Moreover, if $P(g) \neq \emptyset$, we can choose $\varphi$ so that $\varphi(P(\varphi)) \subseteq P(g)$. By construction, $g \circ \varphi: X \rightarrow S^{p}$ is a $\mathcal{C}^{r}$ rational map with $P(g \circ \varphi) \subseteq P(\varphi) \cup \varphi^{-1}(P(g))$, and hence $(g \circ \varphi)(P(g \circ \varphi))$ contains at most one point. Making use of Theorem 2.4, we obtain a $\mathcal{C}^{r}$ rational map $f: X \rightarrow S^{p}$ satisfying the required conditions.

The experience following from $[6,7,8,9]$ shows that in the investigation of regular maps between real algebraic varieties, one frequently encounters $\mathbb{F}$-vector bundles, where $\mathbb{F}$ stands for $\mathbb{R}, \mathbb{C}$ or $\mathbb{H}$ (the quaternions). It turns out that $\mathbb{F}$-vector bundles are useful, and in Section 3 indispensable, for the purposes of the present paper. For any algebraic variety $X$, algebraic $\mathbb{F}$-vector subbundles of the standard trivial $\mathbb{F}$-vector bundle with total space $X \times \mathbb{F}^{n}$, for some $n$, are called algebraic $\mathbb{F}$-vector bundles on $X$. In other words, algebraic $\mathbb{F}$-vector bundles on $X$ correspond to finitely generated projective modules over the ring of regular functions from $X$ into $\mathbb{F}$, cf. [6] for other equivalent definitions. In order to 
prevent any confusion, let us mention that the objects called here and in [6] algebraic $\mathbb{F}$ vector bundles were called in earlier papers $[4,5,7,8,9,11]$ strongly algebraic $\mathbb{F}$-vector bundles. An $\mathbb{F}$-vector bundle on a real algebraic variety is said to admit an algebraic structure if it is isomorphic to an algebraic $\mathbb{F}$-vector bundle (cf. $[5,7,11]$, where this notion is extensively studied). In this section we use exclusively $\mathbb{R}$-vector bundles.

If $p$ is one of the integers $1,2,4$ or 8 , then on $S^{p}$ there is a smooth $\mathbb{R}$-vector bundle $\theta_{p}$ of $\operatorname{rank} p$, which has a smooth section transverse to the zero section and with the zero set consisting precisely of one point (this is certainly well known, but if desired [12, Theorem 1.5], containing a more general fact, can be consulted). It readily follows that for any point $y$ in $S^{p}$, one can find a smooth section $v$ of $\theta_{p}$, which is transverse to the zero section of $\theta_{p}$ and satisfies $Z(v)=\{y\}$, where $Z(v)=\left\{z \in S^{p} \mid v(z)=0\right\}$ is the zero set of $v$.

Corollary 2.7. Let $X$ be a compact nonsingular irreducible real algebraic variety and let $h: X \rightarrow S^{p}$ be a continuous map. Assume that $p$ is one of the integers 1, 2, 4, or 8 , and the $\mathbb{R}$-vector bundle $h^{*} \theta_{p}$ on $X$ admits an algebraic structure. Then for any nonnegative integer $r$, the map $h$ is homotopic to a $\mathcal{C}^{r}$ rational map $f: X \rightarrow S^{p}$ with $\operatorname{codim}_{X} P(f) \geq$ $p$ and $f(P(f))$ containing at most one point. In particular, the conclusion holds if every $\mathbb{R}$-vector bundle of rank $p$ on $X$ admits an algebraic structure.

Proof. We may assume that $h$ is a smooth map. By Sard's Theorem, $h$ is transverse to some point $y$ in $S^{p}$. Hence $P T(h)$ is represented by the framed submanifold $\left(h^{-1}(y)\right.$, $F(h, B)$ ), where $B$ is a positively oriented basis of the tangent space $\tau\left(S^{p}\right)_{y}$.

Let $v: S^{p} \rightarrow \theta_{p}$ be a smooth section transverse to the zero section of $\theta_{p}$ and satisfying $Z(v)=\{y\}$. By assumption, there exist an algebraic $\mathbb{R}$-vector bundle $\xi$ on $X$ and an $\mathbb{R}$-vector bundle isomorphism $\varphi: h^{*} \theta_{p} \rightarrow \xi$. General theory of smooth vector bundles allows us to assume that $\varphi$ is a smooth isomorphism. The smooth section $u:=\varphi \circ\left(h^{*} v\right)$ : $X \rightarrow \xi$ is transverse to the zero section of $\xi$ and $Z(u)=h^{-1}(y)$. One can find an algebraic section $s: X \rightarrow \xi$ close to $u$ in the $\mathcal{C}^{\infty}$ topology, cf. [6, Theorem 12.3.2]. Hence, $Z(s)$ is a nonsingular algebraic subset of $X$ isotopic to $h^{-1}(y)$. It follows that $\left(h^{-1}(y), F(h, B)\right)$ is framed cobordant to $(Z(s), F)$, where $F$ is a suitable framing of $Z(s)$. By Theorem 2.4, there is a $\mathcal{C}^{r}$ rational map $f: X \rightarrow S^{p}$ satisfying the required conditions. The proof is complete.

We shall now derive a consequence of Theorem 2.4 related to the cohomotopy groups. Let $X$ be a compact nonsingular irreducible real algebraic variety and let $p$ be a positive integer. Denote by

$$
\pi_{\text {rat }}^{p}(X)
$$

the subset of $\pi^{p}(X)$ consisting of the homotopy classes $[f]$, where $f: X \rightarrow S^{p}$ is a continuous rational map with $f(P(f))$ containing at most one point. According to Theorem 2.4, for any nonnegative integer $r$, each element of $\pi_{\text {rat }}^{p}(X)$ is the homotopy class of a $\mathcal{C}^{r}$ rational map $f: X \rightarrow S^{p}$ with $\operatorname{codim}_{X} P(f) \geq p$ and $f(P(f))$ containing at most one point. Obviously, $\pi_{\text {rat }}^{p}(X)$ contains the subset $\pi_{\text {alg }}^{p}(X)$ of $\pi^{p}(X)$ consisting of the homotopy classes of regular maps from $X$ into $S^{p}$ (cf. [6, 8] for results concerning $\left.\pi_{\text {alg }}^{p}(X)\right)$. 
If $\operatorname{dim} X \leq 2 p-2$, then $\pi^{p}(X)$ is endowed with the structure of commutative group, called the $p$ th cohomotopy group of $X$, cf. [17]. It is not known whether $\pi_{\text {alg }}^{p}(X)$ is always a subgroup of $\pi^{p}(X)$, cf. [6, p. 361].

Proposition 2.8. With notation as above, if $\operatorname{dim} X \leq 2 p-2$, then $\pi_{\mathrm{rat}}^{p}(X)$ is a subgroup of $\pi^{p}(X)$.

Proof. First we show that $\pi_{\text {rat }}^{p}(X)$ is closed under addition. Let $\left[f_{1}\right]$ and $\left[f_{2}\right]$ be in $\pi_{\text {rat }}^{p}(X)$. We may assume that $f_{i}: X \rightarrow S^{p}$ is a continuous rational map with $\operatorname{codim}_{X} P\left(f_{i}\right) \geq p$ and $f_{i}\left(P\left(f_{i}\right)\right) \subseteq\{b\}$ for some point $b$ in $S^{p}, i=1,2$. Set

$$
A=P\left(f_{1}\right) \cup P\left(f_{2}\right) \quad \text { and } \quad B=f_{1}(A) \cup f_{2}(A) .
$$

Since $\operatorname{dim} P\left(f_{i}\right) \leq \operatorname{dim} X-p \leq p-2$ and $f_{i}$ is a semi-algebraic map for $i=1,2$, we get $\operatorname{dim} B \leq p-2$, cf. [6, Theorem 2.8.8]. By Sard's Theorem, there is a point $\left(y_{1}, y_{2}\right)$ in $\left(S^{p} \backslash B\right) \times\left(S^{p} \backslash B\right)$ such that the restriction of $f_{i}$ to $X \backslash A$ is transverse to $y_{i}$ for $i=1,2$, and the restriction of $\left(f_{1}, f_{2}\right): X \rightarrow S^{p} \times S^{p}$ to $X \backslash A$ is transverse to $\left(y_{1}, y_{2}\right)$. The last condition and the assumption $\operatorname{dim} X \leq 2 p-2$ imply

$$
f_{1}^{-1}\left(y_{1}\right) \cap f_{2}^{-1}\left(y_{2}\right)=\emptyset \text {. }
$$

Choosing a positively oriented basis $B_{i}$ of the tangent space $\tau\left(S^{p}\right)_{y_{i}}$, we obtain that $P T\left(f_{i}\right)$ is represented by $\left(f_{i}^{-1}\left(y_{i}\right), F\left(f_{i}, B_{i}\right)\right)$. The element $\left[f_{1}\right]+\left[f_{2}\right]$ of $\pi^{p}(X)$ corresponds to the framed cobordism class of $(N, F)$, where $N=f_{1}^{-1}\left(y_{1}\right) \cup f_{2}^{-1}\left(y_{2}\right)$ and the restriction of $F$ to $f_{i}^{-1}\left(y_{i}\right)$ is equal to $F\left(f_{i}, B_{i}\right)$ for $i=1,2$. By construction, $N$ is a nonsingular Zariski closed subset of $X \backslash A$. According to Lemma 2.3, $N$ is the set of nonsingular points of its Zariski closure in $X$, which in view of Theorem 2.4 implies that $\left[f_{i}\right]+\left[f_{2}\right]$ is in $\pi_{\text {rat }}^{p}(X)$.

For any $[f]$ in $\pi^{p}(X)$, we have $-[f]=[\varphi \circ f]$, where $\varphi: S^{p} \rightarrow S^{p}$ is an arbitrary continuous map of topological degree -1 . We can choose $\varphi$ regular, and hence $-[f]$ is in $\pi_{\text {rat }}^{p}(X)$, provided $[f]$ is in $\pi_{\text {rat }}^{p}(X)$.

Corollary 2.7 with $p=1$ is contained in Corollary 1.4, which is based on Proposition 1.3 to be proved now. Recall that for any regular map $\varphi: X \rightarrow Y$ between compact nonsingular real algebraic varieties,

$$
\begin{aligned}
\varphi_{*}\left(H_{d}^{\mathrm{alg}}(X, \mathbb{Z} / 2)\right) \subseteq H_{d}^{\mathrm{alg}}(Y, \mathbb{Z} / 2), \\
\varphi^{*}\left(H_{\mathrm{alg}}^{c}(Y, \mathbb{Z} / 2)\right) \subseteq H_{\mathrm{alg}}^{c}(X, \mathbb{Z} / 2)
\end{aligned}
$$

for all $d \geq 0$ and $c \geq 0$, cf. [14, Section 5] or [4].

Given a compact smooth manifold $M$, we denote by $[M]$ its fundamental class in $H_{m}(M, \mathbb{Z} / 2), m=\operatorname{dim} M$. As in Section $1, D_{M}: H^{*}(M, \mathbb{Z} / 2) \rightarrow H_{*}(M, \mathbb{Z} / 2)$ will denote the Poincaré duality isomorphism. 
Proof of Proposition 1.3. By Hironaka's theorem on resolution of points of indeterminacy [16], there is a commutative diagram

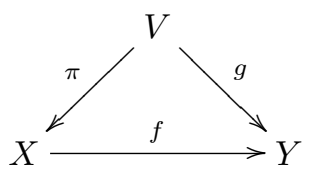

where $\pi: V \rightarrow X$ is a multiblowup of $X$ over $P(f)$ and $g: V \rightarrow Y$ is a regular map. In particular, $V$ is a compact nonsingular real algebraic variety and $\pi_{*}([V])=[X]$.

Given $\beta$ in $H_{d}^{\mathrm{alg}}(X, \mathbb{Z} / 2)$, set $\alpha=D_{V}\left(\pi^{*}\left(D_{X}^{-1}(\beta)\right)\right)$. Since $\pi$ is a regular map, $\alpha$ is in $H_{d}^{\text {alg }}(V, \mathbb{Z} / 2)$. A simple computation involving $\cup$ and $\cap$ products yields

$$
\begin{aligned}
\pi_{*}(\alpha) & =\pi_{*}\left(\pi^{*}\left(D_{X}^{-1}(\beta)\right) \cap[V]\right) \\
& =D_{X}^{-1}(\beta) \cap \pi_{*}([V])=D_{X}^{-1}(\beta) \cap[X]=\beta,
\end{aligned}
$$

and hence

$$
f_{*}(\beta)=f_{*}\left(\pi_{*}(\alpha)\right)=(f \circ \pi)_{*}(\alpha)=g_{*}(\alpha),
$$

which in turn implies that $f_{*}(\beta)$ is in $H_{d}^{\mathrm{alg}}(Y, \mathbb{Z} / 2)$, the map $g$ being regular. Hence

$$
f_{*}\left(H_{d}^{\mathrm{alg}}(X, \mathbb{Z} / 2)\right) \subseteq H_{d}^{\mathrm{alg}}(Y, \mathbb{Z} / 2) .
$$

For any $v$ in $H_{\mathrm{alg}}^{c}(Y, \mathbb{Z} / 2)$, we have $\pi^{*}\left(f^{*}(v)\right)=(f \circ \pi)^{*}(v)=g^{*}(v)$, and hence

$$
\begin{aligned}
D_{X}\left(f^{*}(v)\right) & =f^{*}(v) \cap[X]=f^{*}(v) \cap \pi_{*}([V]) \\
& =\pi_{*}\left(\pi^{*}\left(f^{*}(v)\right) \cap[V]\right)=\pi_{*}\left(g^{*}(v) \cap[V]\right)=\pi_{*}\left(D_{V}\left(g^{*}(v)\right)\right),
\end{aligned}
$$

which in turn implies

$$
f^{*}(v)=D_{X}^{-1}\left(\pi_{*}\left(D_{V}\left(g^{*}(v)\right)\right)\right) .
$$

Since $\pi$ and $g$ are regular maps, it follows that $f^{*}(v)$ is in $H_{\text {alg }}^{c}(X, \mathbb{Z} / 2)$. Hence

$$
f^{*}\left(H_{\text {alg }}^{c}(Y, \mathbb{Z} / 2)\right) \subseteq H_{\text {alg }}^{c}(X, \mathbb{Z} / 2) .
$$

The proof is complete.

As an immediate consequence of Proposition 1.3 we can obtain a result concerning $\mathcal{C}^{r}$ rational maps with values in real projective $q$-space $\mathbb{P}^{q}(\mathbb{R})$, containing Corollary 1.4 as a special case. Recall that $\mathbb{P}^{1}(\mathbb{R})$ is biregularly isomorphic to $S^{1}$.

Corollary 2.9. For any compact nonsingular irreducible real algebraic variety $X$ and any continuous map $h: X \rightarrow \mathbb{P}^{q}(\mathbb{R})$, the following conditions are equivalent:

(a) $h$ is homotopic to a continuous rational map.

(b) $h$ is homotopic to a regular map.

(c) $h$ can be approximated by regular maps. 
(d) $h^{*}(\lambda)$ is in $H_{\text {alg }}^{1}(X, \mathbb{Z} / 2)$, where $\lambda$ is the unique generator of $H^{1}\left(\mathbb{P}^{q}(\mathbb{R}), \mathbb{Z} / 2\right) \cong$ $\mathbb{Z} / 2$.

Proof. It is known that Conditions (b), (c), (d) are equivalent, cf. [6, Theorems 12.4.6 and 13.3.1]. Since $H^{1}\left(\mathbb{P}^{q}(\mathbb{R}), \mathbb{Z} / 2\right)=H_{\text {alg }}^{1}\left(\mathbb{P}^{q}(\mathbb{R}), \mathbb{Z} / 2\right)$, by Proposition 1.3, (a) implies (d), while (b) trivially implies (a).

We conclude this section by proving Theorem 1.5. First some preparation is necessary. If $N$ is a compact smooth submanifold of a smooth manifold $M$, we denote by $[N]_{M}$ the homology class represented by $N$ in $H_{n}(M, \mathbb{Z} / 2), n=\operatorname{dim} N$. In other words, $[N]_{M}=i_{*}([N])$, where $i: N \hookrightarrow M$ is the inclusion map. As usual, $w_{1}(\xi)$ will stand for the first Stiefel-Whitney class of an $\mathbb{R}$-vector bundle $\xi$, and $w_{1}(M)=w_{1}(\tau(M))$. By a smooth curve we mean a smooth manifold of dimension 1.

Let $Y$ be a compact nonsingular real algebraic variety. An algebraic curve in $Y$ is, by definition, an algebraic subset of $Y$ of dimension 1. We say that a smooth curve $C$ in $Y$ can be approximated by nonsingular algebraic curves in $Y$ if every neighborhood of the inclusion map $C \hookrightarrow Y$ (in the $\mathcal{C}^{\infty}$ topology) contains a smooth embedding $e: C \rightarrow Y$ such that $e(C)$ is a nonsingular algebraic curve in $Y$. A characterization of smooth curves $C$ in $Y$ having this approximation property will be needed. It is derived for curves with trivial normal bundle since a more general result is not required in the present paper (cf. Lemma 2.15). Results of [1] will be freely used.

Notation 2.10. In the remaining part of this section $V$ will denote a compact nonsingular real algebraic variety with $\operatorname{dim} V \geq 3$.

Lemma 2.11. Let $C$ be a smooth curve in $V$ and let $A$ be a nonsingular algebraic curve in $V$. Assume that $A \cap C=\emptyset$ and $A \cup C=\partial F$, where $F$ is a compact smooth surfacewith-boundary in $V$ with trivial normal bundle. Then $C$ can be approximated by nonsingular algebraic curves in $V$.

Proof. Set $\operatorname{dim} V=n+1$. There is a smooth map $f: V \rightarrow \mathbb{R}^{n}$ transverse to 0 in $\mathbb{R}^{n}$ and with $f^{-1}(0)=A \cup C$, cf. [12, Theorem 1.12]. By the relative Weierstrass approximation theorem [6, Lemma 12.5.5], one can find a regular map $g: V \rightarrow \mathbb{R}^{n}$ close to $f$ in the $\mathcal{C}^{\infty}$ topology and satisfying $A \subseteq g^{-1}(0)$. If $g$ is sufficiently close to $f$, then $g$ is transverse to 0 , and hence $g^{-1}(0)$ is a nonsingular algebraic curve in $V$. Moreover, $g^{-1}(0)=A \cup B$ with $A \cap B=\emptyset$, which implies that $B$ is a nonsingular algebraic curve in $V$, cf. [6, Proposition 3.3.17]. By construction, $B$ approximates $C$.

Lemma 2.12. Let $C$ be a smooth curve in $V$. Assume that for each connected component $D$ of $C$, the modulo 2 intersection number of $[D]_{V}$ and $D_{V}\left(w_{1}(V)\right)$ is zero. Then there is a smooth submanifold $S$ of $V$ satisfying $\operatorname{codim}_{V} S=1, D_{V}\left(w_{1}(V)\right)=[S]_{V}$, and $S \cap C=\emptyset$.

Proof. Let $N$ be any smooth submanifold of $V$ with $\operatorname{codim}_{V} N=1$ and $D_{V}\left(w_{1}(V)\right)=$ $[N]_{V}$. Note that $N \cap D$ consists of an even number of points for every connected component $D$ of $C$. We obtain $S$ with the required properties by modifying $N$ as in $[1, \mathrm{p} .213]$ or [10, p. 599]. 
Lemma 2.13. Let $C$ be a smooth curve in $V$. Let $S$ be a smooth submanifold of $V$ with $\operatorname{codim}_{V} S=1$ and $D_{V}\left(w_{1}(V)\right)=[S]_{V}$. If $i: V \backslash S \hookrightarrow V$ is the inclusion map, then every homology class in the kernel of the induced homomorphism

$$
i_{*}: H_{1}(V \backslash S, \mathbb{Z} / 2) \rightarrow H_{1}(V, \mathbb{Z} / 2)
$$

is of the form $[B]_{V \backslash S}$, where $B$ is a nonsingular algebraic curve in $V, B \subseteq V \backslash S$, and $B \cap C=\emptyset$.

Proof. Denoting by $\nu(S)$ the normal bundle of $S$ in $V$, we have $\tau(S) \oplus \nu(S) \cong \tau(V) \mid S$. Hence $w_{1}(S)+w_{1}(\nu(S))=j^{*}\left(w_{1}(V)\right)$, where $j: S \hookrightarrow V$ is the inclusion map. Since $D_{V}\left(w_{1}(V)\right)=[S]_{V}$, we get $j^{*}\left(w_{1}(V)\right)=w_{1}(\nu(S))$, cf. [20, Theorem 11.3]. Therefore $w_{1}(S)=0$, which means that $S$ is orientable. One completes the proof (the essential part of it) by repeating the argument used in [1, Lemma 5].

Lemma 2.14. Let $A$ be a nonsingular algebraic curve in $V$. Then there is a nonsingular algebraic curve $B$ in $V$ with $[A]_{V}=[B]_{V}$ and such that each connected component of $V$ contains at most one connected component of $B$.

Proof. It suffices to repeat the argument used in [1, Lemma 6].

The last four lemmas are needed only to prove the next one, which in turn will be used in the proof of Theorem 1.5.

Lemma 2.15. Let $K$ be a smooth curve in $V$ with trivial normal bundle. Assume $[K]_{V}=$ $[A]_{V}$, where $A$ is a nonsingular algebraic curve in $V$. Then $K$ can be approximated by nonsingular algebraic curves in $V$.

Proof. In virtue of Lemma 2.14, we may assume that each connected component of $V$ contains at most one connected component of $A$. Applying a small isotopy to $K$ and making use of transversality, we may assume $K \cap A=\emptyset$.

We will now verify that Lemma 2.12 (with $C=K \cup A$ ) is applicable.

Let $L$ be a connected component of $K$. Since the vector bundles $\tau(L)$ and $\nu(L)$ are trivial, and $\tau(L) \oplus \nu(L) \cong \tau(V) \mid L$, we get

$$
j^{*}\left(w_{1}(V)\right)=0
$$

where $j: L \hookrightarrow V$ is the inclusion map. Hence a simple computation yields

$$
\begin{aligned}
\left\langle w_{1}(V) \cup D_{V}^{-1}\left([L]_{V}\right),[V]\right\rangle & =\left\langle w_{1}(V), D_{V}^{-1}\left([L]_{V}\right) \cap[V]\right\rangle=\left\langle w_{1}(V),[L]_{V}\right\rangle \\
& =\left\langle w_{1}(V), j_{*}([L])\right\rangle=\left\langle j^{*}\left(w_{1}(V)\right),[L]\right\rangle=0,
\end{aligned}
$$

which means that the modulo 2 intersection number of $[L]_{V}$ and $D_{V}\left(w_{1}(V)\right)$ is zero.

If $H$ is a connected component of $A$, then either $[H]_{V}=0$ or $[H]_{V}=\left[K^{\prime}\right]_{V}$, where $K^{\prime}$ is the union of some connected components of $K$. Thus the modulo 2 intersection number of $[H]_{V}$ and $D_{V}\left(w_{1}(V)\right)$ is zero. 
Hence by Lemma 2.12, there is a smooth submanifold $S$ in $V$ with $\operatorname{codim}_{V} S=1$, $D_{V}\left(w_{1}(V)\right)=[S]_{V}$, and $S \cap(K \cup A)=\emptyset$. Let $i: V \backslash S \hookrightarrow V$ be the inclusion map and let

$$
i_{*}: H_{1}(V \backslash S, \mathbb{Z} / 2) \rightarrow H_{1}(V, \mathbb{Z} / 2)
$$

be the induced homomorphism. Since $[K]_{V \backslash S}-[A]_{V \backslash S}$ is in the kernel of $i_{*}$, it follows from Lemma 2.13 (with $C=K \cup A$ ) that $[K]_{V \backslash S}-[A]_{V \backslash S}=[B]_{V \backslash S}$, or equivalently,

$$
[K]_{V \backslash S}+[A]_{V \backslash S}+[B]_{V \backslash S}=0
$$

for some nonsingular algebraic curve $B$ in $V$ satisfying $B \subseteq V \backslash S$ and $B \cap(K \cup A)=\emptyset$.

The equality $D_{V}\left(w_{1}(V)\right)=[S]_{V}$ implies that $V \backslash S$ is an orientable smooth manifold. In particular, every smooth curve in $V \backslash S$ has trivial normal bundle. For any oriented smooth curve $E$ in $V \backslash S$, denote by $o_{E}$ its homology class in $H_{1}(V \backslash S, \mathbb{Z})$. Endowing the curves $K, A, B$ with orientations, we get

$$
o_{K}+o_{A}+o_{B}+2 v=0
$$

for some homology class $v$ in $H_{1}(V \backslash S, \mathbb{Z})$. Let $D_{0}$ be an oriented smooth curve in $V \backslash S$ with $o_{D_{0}}=v$ and $D_{0} \cap(K \cup A \cup B)=\emptyset$. There is a smooth embedding $e: D_{0} \times[0,1] \rightarrow$ $V \backslash S$ such that the smooth surface-with-boundary $G=e\left(D_{0} \times[0,1]\right)$ has trivial normal bundle, $e\left(D_{0} \times\{0\}\right)=D_{0}$, and $G \cap(K \cup A \cup B)=\emptyset$. Note that $\partial G=D_{0} \cup D_{1}$, where $D_{1}=e\left(D_{0} \times\{1\}\right)$. Endowing $D_{1}$ with an appropriate orientation, we have $o_{D_{1}}=v$. By Lemma 2.11, there is a nonsingular algebraic curve $C$ in $Y$ which approximates $D_{0} \cup D_{1}$. We can choose $C$ satisfying $C \subseteq X \backslash S$ and $C \cap(K \cup A \cup B)=\emptyset$. Orienting $C$ in a suitable way, we get $o_{C}=2 v$. Hence regarding $K \cup A \cup B \cup C$ as an oriented smooth curve in $V \backslash S$, we obtain

$$
o_{K \cup A \cup B \cup C}=0 .
$$

It follows that $K \cup A \cup B \cup C=\partial F$ for some compact oriented smooth surface-withboundary $F$ in $V \backslash S$. We can discard the connected components of $F$ with empty boundary. Then the orientability of $F$ implies that its normal bundle is trivial. Since $A \cup B \cup C$ is a nonsingular algebraic curve in $V$, we complete the proof by applying Lemma 2.11 .

The next observation is of a different nature.

Lemma 2.16. Let $D$ be a smooth curve in $V$ and let $B$ be an algebraic (possibly singular) curve in $V$ satisfying $D \cap B=\emptyset$ and $[D]_{V}=[B]_{V}$. Let $\rho: W \rightarrow V$ be the blowup of $V$ at a point $b$ in $V \backslash D$. If $C=\rho^{-1}(D)$ and $A$ is the strict transform of $B$ under $\rho$, then

$$
[C]_{W}=[A]_{W}+[H]_{W},
$$

where $H$ is a nonsingular algebraic curve in $W$ satisfying $H \subseteq \rho^{-1}(b)$ and $A \cap H=\emptyset$.

Proof. Set $E=\rho^{-1}(b)$. The homology class $[C]_{W}-[A]_{W}$ is in the kernel of $\rho_{*}$, and hence

$$
[C]_{W}=[A]_{W}+j_{*}(v),
$$


where $v$ is in $H_{1}(E, \mathbb{Z} / 2)$ and $j: E \hookrightarrow W$ is the inclusion map (cf. for example [3, Lemma 2.9.3]). If $\operatorname{dim} V=n+1$, then $E$ is biregularly isomorphic to $\mathbb{P}^{n}(\mathbb{R})$. Since $n \geq 2$ and $A \cap E$ is a finite set, we have $j_{*}(v)=[H]_{W}$ with $H$ satisfying the required conditions.

Proof of Theorem 1.5. Clearly, (a) implies (b), and in view of Proposition 1.3, part (b) implies (c).

Assuming that (c) is satisfied, we will prove now that (a) holds. If $n=1$, then (a) follows from Corollary 1.4, and therefore in what follows $n \geq 2$. Without loss of generality, we may assume $h$ to be a smooth map.

Let $B$ be an algebraic (possibly singular) curve in $X$ with $[B]_{X}$ Poincaré dual to $h^{*}\left(\sigma_{n}\right)$. There is a sequence of blowups

$$
Y=X_{k} \stackrel{\pi_{k}}{\longrightarrow} X_{k-1} \stackrel{\pi_{k-1}}{\longrightarrow} \cdots \stackrel{\pi_{2}}{\longrightarrow} X_{1} \stackrel{\pi_{1}}{\longrightarrow} X_{0}=X,
$$

each blowup at one point, such that the composite map $\pi=\pi_{1} \circ \cdots \circ \pi_{k}: Y \rightarrow X$ is a multiblowup of $X$ over the set $S$ of singular points of $B$, and the strict transform $A$ of $B$ under $\pi$ is a nonsingular algebraic curve in $Y$.

By Sard's Theorem, $h$ is transverse to some point $y$ in $S^{n} \backslash h(B)$. Thus $D=h^{-1}(y)$ is a smooth curve in $X$ with $D \cap B=\emptyset$. Since the normal bundle of $D$ in $X$ is trivial and the restriction $\pi_{S}: Y \backslash \pi^{-1}(S) \rightarrow X \backslash S$ of $\pi$ is a biregular isomorphism, it follows that the smooth curve $K=\pi^{-1}(D)$ in $Y$ has trivial normal bundle. Moreover, in view of Lemma 2.16,

$$
[K]_{Y}=[A]_{Y}+[H]_{Y},
$$

where $H$ is a nonsingular algebraic curve in $Y$ with $H \cap A=\emptyset$. Hence by Lemma 2.15, there is a nonsingular algebraic curve $C$ in $Y$ approximating $K$. We may choose $C \subseteq$ $Y \backslash \pi^{-1}(S)$. Thus $\pi(C)$ is a smooth curve in $X$ isotopic to $D$. Moreover, $\pi(C)$ is a nonsingular Zariski closed subset of $X \backslash S$. By Lemma 2.3, $\pi(C)$ is precisely the set of nonsingular points of its Zariski closure in $X$. In virtue of Theorem 2.4, (a) holds. The proof is complete.

\section{Rational maps into Grassmannians}

Let $\mathbb{F}$ stand for $\mathbb{R}, \mathbb{C}$ or $\mathbb{H}$. When convenient we identify $\mathbb{F}$ with $\mathbb{R}^{d(\mathbb{F})}$, where $d(\mathbb{F})=$ $\operatorname{dim}_{\mathbb{R}} \mathbb{F}$. Denote by $\mathbb{G}_{n, p}(\mathbb{F})$ the Grassmannian of $p$-dimensional vector subspaces of $\mathbb{F}^{n}$. As in $[5,6,7]$, we shall always regard $\mathbb{G}_{n, p}(\mathbb{F})$ as a real algebraic variety. The universal $\mathbb{F}$-vector bundle $\gamma_{n, p}(\mathbb{F})$ on $\mathbb{G}_{n, p}(\mathbb{F})$ is algebraic. In this section we study $\mathcal{C}^{r}$ rational maps with values in $\mathbb{G}_{n, p}(\mathbb{F})$. The main result is Theorem 3.7. In our considerations $\mathbb{F}$ vector bundles will play a crucial role. Given a topological space $Y$, we denote by $\varepsilon_{Y}^{k}(\mathbb{F})$ the standard trivial $\mathbb{F}$-vector bundle on $Y$ with total space $Y \times \mathbb{F}^{k}$. If $\xi$ is an $\mathbb{F}$-vector subbundle of $\varepsilon_{Y}^{k}(\mathbb{F})$, then $\xi^{\perp}$ will stand for the orthogonal complement of $\xi$ with respect to the standard scalar product $\mathbb{F}^{k} \times \mathbb{F}^{k} \rightarrow \mathbb{F}$; thus $\xi^{\perp}$ is an $\mathbb{F}$-vector subbundle of $\varepsilon_{Y}^{k}(\mathbb{F})$ and $\xi \oplus \xi^{\perp}=\varepsilon_{Y}^{k}(\mathbb{F})$. If $Y$ is a real algebraic variety and $\xi$ is an algebraic $\mathbb{F}$-vector subbundle of $\varepsilon_{Y}^{k}(\mathbb{F})$, then $\xi^{\perp}$ is also an algebraic $\mathbb{F}$-vector subbundle of $\varepsilon_{Y}^{k}(\mathbb{F})$. 
Notation 3.1. Throughout this section $X$ will denote a compact nonsingular irreducible real algebraic variety. Unless explicitly specified otherwise, $r$ will stand for a nonnegative integer or $\infty$.

The following notion will play a key role.

Definition 3.2. An $\mathbb{F}$-vector bundle $\xi$ on $X$ is said to admit a $\mathcal{C}^{r}$ rational structure if there exist a $\mathcal{C}^{r} \mathbb{F}$-vector subbundle $\eta$ of $\varepsilon_{X}^{k}(\mathbb{F})$, for some $k$, and a nonempty Zariski open subset $U$ of $X$ such that $\xi$ is isomorphic to $\eta$ and the restriction $\eta \mid U$ is an algebraic $\mathbb{F}$-vector subbundle of $\varepsilon_{U}^{k}(\mathbb{F})$.

By shrinking $U$, we may require in Definition 3.2 that $\eta \mid U$ be algebraically trivial. Since $U$ is dense in $X$, it follows that $\xi$ has constant rank on $X$.

Of course, every $\mathbb{F}$-vector bundle on $X$ admitting an algebraic structure admits also a $\mathcal{C}^{r}$ rational structure.

Although Definition 3.2 has a somewhat technical character, we will see below that it is "natural". For any ring $A$ (associative with 1 ) denote by $\operatorname{Proj}(A)$ the set of isomorphism classes of finitely generated projective (left) $A$-modules. Denote by $\mathcal{C}(X, \mathbb{F})$ the ring of all continuous functions from $X$ into $\mathbb{F}$. For any $\mathbb{F}$-vector bundle $\xi$ on $X$, the $\mathcal{C}(X, \mathbb{F})$ module $\Gamma(\xi)$ of all continuous sections of $\xi$ is finitely generated and projective. By [23], the correspondence $\xi \rightarrow \Gamma(\xi)$ gives rise to a bijection

$$
V B_{\mathbb{F}}(X) \rightarrow \operatorname{Proj}(\mathcal{C}(X, \mathbb{F})),
$$

where $V B_{\mathbb{F}}(X)$ is the set of isomorphism classes of $\mathbb{F}$-vector bundles on $X$. We regard the ring $\mathcal{R}^{r}(X, \mathbb{F})$ of all $\mathcal{C}^{r}$ rational functions from $X$ into $\mathbb{F}$ as a subring of $\mathcal{C}(X, \mathbb{F})$. According to [24, Theorem 2.2], the map

$$
\operatorname{Proj}\left(\mathcal{R}^{r}(X, \mathbb{F})\right) \rightarrow \operatorname{Proj}(\mathcal{C}(X, \mathbb{F}))
$$

determined by the correspondence $M \rightarrow \mathcal{C}(X, \mathbb{F}) \otimes M$ is injective. The image of this map can be described in terms of $\mathbb{F}$-vector bundles admitting a $\mathcal{C}^{r}$ rational structure, cf. Proposition 3.3. In view of Proposition 2.1, $\mathcal{R}^{\infty}(X, \mathbb{F})$ is the ring of all regular functions from $X$ into $\mathbb{F}$, which is usually denoted by $\mathcal{R}(X, \mathbb{F})$. By [6, Proposition 2.1.12], an $\mathbb{F}$ vector bundle $\xi$ on $X$ admits an algebraic structure if and only if $\Gamma(\xi)$ is isomorphic to $\mathcal{C}(X, \mathbb{F}) \otimes P$ for some finitely generated projective $\mathcal{R}(X, \mathbb{F})$-module $P$.

Proposition 3.3. For any $\mathbb{F}$-vector bundle $\xi$ on $X$ of rank $p$, the following conditions are equivalent:

(a) $\xi$ admits a $\mathcal{C}^{r}$ rational structure.

(b) $\xi$ is isomorphic to $f^{*} \gamma_{n, p}(\mathbb{F})$, where $n \geq p$ and $f: X \rightarrow \mathbb{G}_{n, p}(\mathbb{F})$ is a $\mathcal{C}^{r}$ rational map.

(c) $\Gamma(\xi)$ is isomorphic to $\mathcal{C}(X, \mathbb{F}) \otimes M$ for some finitely generated projective $\mathcal{R}^{r}(X, \mathbb{F})$ module $M$. 
Proof. Assume that (a) holds. Let $\eta$ and $U$ be as in Definition 3.2. Define $g: X \rightarrow$ $\mathbb{G}_{k, p}(\mathbb{F})$ by

$$
\{x\} \times g(x)=\text { the fiber of } \eta \text { over } x \subseteq\{x\} \times \mathbb{F}^{k}
$$

for all $x$ in $X$. Then $g$ is a $\mathcal{C}^{r}$ map and $\eta=g^{*} \gamma_{k, p}(\mathbb{F})$. Thus $\xi$ is isomorphic to $g^{*} \gamma_{k, p}(\mathbb{F})$. Since $g \mid U: U \rightarrow \mathbb{G}_{k, p}(\mathbb{F})$ is a regular map (cf. [6, Proposition 3.4.7]), (b) holds with $n=k$ and $f=g$.

We shall now prove that (a) implies (c). Since $\eta \oplus \eta^{\perp}=\varepsilon_{X}^{k}(\mathbb{F})$, we have $P \oplus P^{\perp} \cong$ $\mathcal{R}^{r}(X, \mathbb{F})^{k}$, where $P$ (respectively $P^{\perp}$ ) is the $\mathcal{R}^{r}(X, \mathbb{F})$-module of all $\mathcal{C}^{r}$ sections of $\eta$ (respectively $\eta^{\perp}$ ) that are $\mathcal{C}^{r}$ rational maps from $U$ into the total space of $\eta \mid U$ (respectively $\left.\eta^{\perp} \mid U\right)$. It follows that $P$ is a finitely generated projective $\mathcal{R}^{r}(X, \mathbb{F})$-module. Moreover, the $\mathcal{C}(X, \mathbb{F})$-modules $\mathcal{C}(X, \mathbb{F}) \otimes P$ and $\Gamma(\eta)$ are isomorphic. Since $\Gamma(\xi)$ is isomorphic to $\Gamma(\eta)$, Condition (c) holds with $M=P$.

Assume now that (b) is satisfied. Note that $f^{*} \gamma_{n, p}(\mathbb{F})$ is a $\mathcal{C}^{r} \mathbb{F}$-vector subbundle of $\varepsilon_{X}^{n}(\mathbb{F})$. Let $V$ be a nonempty Zariski open subset of $X$ for which the restriction $f \mid V$ : $V \rightarrow \mathbb{G}_{n, p}(\mathbb{F})$ is a regular map. Then $\left(f^{*} \gamma_{n, p}(\mathbb{F})\right) \mid V=(f \mid V)^{*} \gamma_{n, p}(\mathbb{F})$ is an algebraic $\mathbb{F}$-vector subbundle of $\varepsilon_{V}^{n}(\mathbb{F})$, and hence (a) holds.

It remains to prove that (c) implies (a). Assume that (c) holds. Let $N$ be an $\mathcal{R}^{r}(X, \mathbb{F})$ module such that $M \oplus N$ is isomorphic to $\mathcal{R}(X, \mathbb{F})^{n}$ for some $n$. Without loss of generality we may assume that $M$ and $N$ are submodules of $\mathcal{R}(X, \mathbb{F})^{n}$ and

$$
M \oplus N=\mathcal{R}^{r}(X, \mathbb{F})^{n} .
$$

Note that the submodule $\bar{M}:=\mathcal{C}(X, \mathbb{F}) M$ of $\mathcal{C}(X, \mathbb{F})^{n}$ is isomorphic to $\mathcal{C}(X, \mathbb{F}) \otimes M$. We complete the proof by constructing an $\mathbb{F}$-vector subbundle $\zeta$ of $\varepsilon_{X}^{n}(\mathbb{F})$ corresponding to $\bar{M}$. Define a subset $E$ of $X \times \mathbb{F}^{n}$ and a map $\pi: E \rightarrow X$ by

$$
\begin{aligned}
& E=\left\{(x, v) \in X \times \mathbb{F}^{n} \mid v=\left(a_{1}(x), \ldots, a_{n}(x)\right) \text { for some }\left(a_{1}, \ldots, a_{n}\right) \text { in } \bar{M}\right\}, \\
& \pi(x, v)=x .
\end{aligned}
$$

The fiber $E_{x}=\pi^{-1}(x)$ of $\pi$ over $x$ is a vector subspace of $\{x\} \times \mathbb{F}^{n}$. In particular, $\zeta:=(E, \pi, X)$ is a family of $\mathbb{F}$-vector spaces on $X$. Since $\bar{M}$ is isomorphic to $\Gamma(\xi)$ and rank $\xi=p$, we have $\operatorname{dim}_{\mathbb{F}} E_{x}=p$ for all $x$ in $X$.

Let $\left[a_{i j}\right]$ be an $m \times n$ matrix with entries in $\mathcal{R}^{r}(X, \mathbb{F})$, whose rows generate $M$ as an $\mathcal{R}^{r}(X, \mathbb{F})$-module. Then $E_{x}=\{x\} \times A_{x}$, where $A_{x}$ is the vector subspace of $\mathbb{F}^{n}$ generated by the rows of the matrix $\left[a_{i j}(x)\right]$. It follows that the map

$$
h: X \rightarrow \mathbb{G}_{n, p}(\mathbb{F}), h(x)=A_{x} \text { for all } x \text { in } X
$$

is well defined and of class $\mathcal{C}^{r}$. By construction, $h^{*} \gamma_{n, p}(\mathbb{F})=\zeta$, which implies that $\zeta$ is a $\mathcal{C}^{r} \mathbb{F}$-vector subbundle of $\varepsilon_{X}^{n}(\mathbb{F})$. If $W$ is a nonempty Zariski open subset of $X$ for which the restrictions $a_{i j} \mid W: W \rightarrow \mathbb{F}$ are regular functions for all $i$ and $j$, then $h \mid W: W \rightarrow$ $\mathbb{G}_{n, p}(\mathbb{F})$ is a regular map (cf. [6, Proposition 3.4.7]) and hence $\zeta \mid W=(h \mid W)^{*} \gamma_{n, p}(\mathbb{F})$ is an algebraic $\mathbb{F}$-vector subbundle of $\varepsilon_{W}^{n}(\mathbb{F})$. Since $\Gamma(\zeta)=\bar{M} \cong \Gamma(\xi)$, it follows that $\xi$ is isomorphic to $\zeta$. Thus $\xi$ admits a $\mathcal{C}^{r}$ rational structure and (a) holds.

Corollary 3.4. An $\mathbb{F}$-vector bundle on $X$ admits a $\mathcal{C}^{r}$ rational structure if and only if it is stably equivalent to an $\mathbb{F}$-vector bundle admitting a $\mathcal{C}^{r}$ rational structure. 
Proof. The assertion follows from Proposition 3.3 and [24, Theorem 2.2].

Corollary 3.5. For any $\mathbb{F}$-vector bundle $\xi$ on $X$, the following conditions are equivalent:

(a) $\xi$ admits a $\mathcal{C}^{\infty}$ rational structure.

(b) $\xi$ admits an algebraic structure.

Proof. Since $\mathcal{R}^{\infty}(X, \mathbb{F})=\mathcal{R}(X, \mathbb{F})$ is the ring of regular functions from $X$ into $\mathbb{F}$, in virtue of Proposition 3.3, (a) implies (b). It is obvious that (b) implies (a).

Any $\mathbb{F}$-vector bundle $\xi$ can be regarded as an $\mathbb{R}$-vector bundle; to indicate that we write $\xi_{\mathbb{R}}$. If $\xi$ admits a $\mathcal{C}^{r}$ rational structure, then so does $\xi_{\mathbb{R}}$. Our next result shows that Definition 3.2 imposes severe restrictions on $\mathbb{F}$-vector bundles.

Theorem 3.6. Let $\xi$ be an $\mathbb{F}$-vector bundle on $X$ admitting a $\mathcal{C}^{r}$ rational structure. Then the kth Stiefel-Whitney class $w_{k}\left(\xi_{\mathbb{R}}\right)$ of $\xi_{\mathbb{R}}$ is in $H_{\mathrm{alg}}^{k}(X, \mathbb{Z} / 2)$ for all $k \geq 0$.

Proof. Let $p=\operatorname{rank} \xi_{\mathbb{R}}$. By Proposition 3.3, there is an integer $n \geq p$ and a $\mathcal{C}^{r}$ rational map $f: X \rightarrow \mathbb{G}_{n, p}(\mathbb{R})$ with $f^{*} \gamma_{n, p}(\mathbb{R}) \cong \xi_{\mathbb{R}}$. This implies $w_{k}\left(\xi_{\mathbb{R}}\right)=f^{*}\left(w_{k}\left(\gamma_{n, p}(\mathbb{R})\right)\right)$. Since

$$
H_{\text {alg }}^{k}\left(\mathbb{G}_{n, p}(\mathbb{R}), \mathbb{Z} / 2\right)=H^{k}\left(\mathbb{G}_{n, p}(\mathbb{R}), \mathbb{Z} / 2\right)
$$

(cf. [6, Proposition 11.3.3]), it follows from Proposition 1.3 that $w_{k}\left(\xi_{\mathbb{R}}\right)$ is in $H_{\mathrm{alg}}^{k}(X$, $\mathbb{Z} / 2$ ).

Theorem 3.7. For any $\mathcal{C}^{r}$ map $h: X \rightarrow \mathbb{G}_{n, p}$, the following conditions are equivalent:

(a) $h$ can be approximated in the $\mathcal{C}^{r}$ topology by $\mathcal{C}^{r}$ rational maps.

(b) $h$ is homotopic to a $\mathcal{C}^{r}$ rational map.

(c) The pullback $\mathbb{F}$-vector bundle $h^{*} \gamma_{n, p}(\mathbb{F})$ on $X$ admits a $\mathcal{C}^{r}$ rational structure.

Proof. In order to ease notation, we will write $\mathbb{G}_{n, p}, \gamma_{n, p}, \varepsilon_{Y}^{i}$ instead of $\mathbb{G}_{n, p}(\mathbb{F}), \gamma_{n, p}(\mathbb{F})$, $\varepsilon_{Y}^{i}(\mathbb{F})$, respectively. Obviously, (a) implies (b). If (b) holds and $h$ is homotopic to a $\mathcal{C}^{r}$ rational map $g: X \rightarrow \mathbb{G}_{n, p}$, then the $\mathbb{F}$-vector bundles $h^{*} \gamma_{n, p}$ and $g^{*} \gamma_{n, p}$ are isomorphic, and hence (c) is satisfied in virtue of Proposition 3.3.

It remains to prove that (c) implies (a). Assume (c) holds and $h^{*} \gamma_{n, p}$ is isomorphic to a $\mathcal{C}^{r} \mathbb{F}$-vector subbundle $\eta$ of $\varepsilon_{X}^{k}$, whose restriction $\eta \mid U$ to a nonempty Zariski open subset $U$ of $X$ is an algebraic $\mathbb{F}$-vector subbundle of $\varepsilon_{U}^{k}$. Since $\eta$ and $h^{*} \gamma_{n, p}$ are $\mathcal{C}^{r} \mathbb{F}$-vector bundles, there is a $\mathcal{C}^{r} \mathbb{F}$-vector bundle isomorphism $\varphi: \eta \rightarrow h^{*} \gamma_{n, p}$. Regarding $h^{*} \gamma_{n, p}$ as a subbundle of $\varepsilon_{X}^{n}$, we define a $\mathcal{C}^{r}$ section $w: X \rightarrow \operatorname{Hom}\left(\eta, \varepsilon_{X}^{n}\right)$ by $w(x)(e)=\varphi(e)$ for all $x$ in $X$ and $e$ in the fiber $E(\eta)_{x}$ of $\eta$ over $x$. Note that $w(x): E(\eta)_{x} \rightarrow\{x\} \times \mathbb{F}^{n}$ is an injective $\mathbb{F}$-linear transformation for all $x$ in $X$.

Claim. There is a $\mathcal{C}^{r}$ section $s: X \rightarrow \operatorname{Hom}\left(\eta, \varepsilon_{X}^{n}\right)$, arbitrarily close to $w$ in the $\mathcal{C}^{r}$ topology, such that $s \mid U$ is an algebraic section of $\operatorname{Hom}\left(\eta, \varepsilon_{X}^{n}\right) \mid U=\operatorname{Hom}\left(\eta \mid U, \varepsilon_{U}^{n}\right)$. 
The equality $\varepsilon_{X}^{k}=\eta \oplus \eta^{\perp}$ implies the existence of a $\mathcal{C}^{r}$ section $v: X \rightarrow \operatorname{Hom}\left(\varepsilon_{X}^{k}\right.$, $\left.\varepsilon_{X}^{n}\right)$ satisfying $w=\rho \circ v$, where

$$
\rho: \operatorname{Hom}\left(\varepsilon_{X}^{k}, \varepsilon_{X}^{n}\right) \rightarrow \operatorname{Hom}\left(\eta, \varepsilon_{X}^{n}\right), \rho(\psi)=\psi \mid \eta
$$

is a $\mathcal{C}^{r}$ homomorphism of $\mathbb{F}$-vector bundles. Sections of $\operatorname{Hom}\left(\varepsilon_{X}^{k}, \varepsilon_{X}^{n}\right)$ can be identified with maps from $X$ into $\mathbb{F}^{k n}$, and hence by the Weierstrass approximation theorem, there exists an algebraic section $u: X \rightarrow \operatorname{Hom}\left(\varepsilon_{X}^{k}, \varepsilon_{X}^{n}\right)$ close to $v$ in the $\mathcal{C}^{r}$ topology. Thus the $\mathcal{C}^{r}$ section $s=\rho \circ u: X \rightarrow \operatorname{Hom}\left(\eta, \varepsilon_{X}^{n}\right)$ is close to $w$. Since $\eta \mid U$ and $\eta^{\perp} \mid U$ are algebraic $\mathbb{F}$-vector subbundles of $\varepsilon_{U}^{k}$, the restriction

$$
\operatorname{Hom}\left(\varepsilon_{X}^{k}, \varepsilon_{X}^{n}\right)\left|U=\operatorname{Hom}\left(\varepsilon_{U}^{k}, \varepsilon_{U}^{n}\right) \rightarrow \operatorname{Hom}\left(\eta, \varepsilon_{X}^{n}\right)\right| U=\operatorname{Hom}\left(\eta \mid U, \varepsilon_{U}^{n}\right)
$$

of $\rho$ is an algebraic homomorphism. It follows that $s \mid U$ is an algebraic section. The claim is proved.

If $s$ is sufficiently close to $w$, then $s(x): E(\eta)_{x} \rightarrow\{x\} \times \mathbb{F}^{n}$ is an injective $\mathbb{F}$-linear transformation for all $x$ in $X$. The map $f: X \rightarrow \mathbb{G}_{n, p}$, defined by $\{x\} \times f(x)=$ $s(x)\left(E(\eta)_{x}\right)$ for all $x$ in $X$, is of class $\mathcal{C}^{r}$ and its restriction $f \mid U$ is a regular map, cf. [6, Proposition 3.4.7]. Moreover, $f$ is close to $h$, provided $s$ is close to $w$. Hence (a) holds.

Of particular interest is Theorem 3.7 for maps with values in $\mathbb{G}_{2,1}(\mathbb{F})$. Recall that $\mathbb{G}_{2,1}(\mathbb{F})$ is biregularly isomorphic to $S^{d(\mathbb{F})}$, where $d(\mathbb{F})=\operatorname{dim}_{\mathbb{R}}(\mathbb{F})$. If $a=(0,1) \in \mathbb{F} \times \mathbb{R}$ and if $\rho: S^{d(\mathbb{F})} \backslash\{a\} \rightarrow \mathbb{R}^{d(\mathbb{F})}=\mathbb{F}$ is the sterographic projection, then $\alpha_{\mathbb{F}}: S^{d(\mathbb{F})} \rightarrow$ $\mathbb{G}_{2,1}(\mathbb{F})$, defined by

$$
\alpha_{\mathbb{F}}(x)= \begin{cases}\mathbb{F}(\rho(x), 1) \subseteq \mathbb{F}^{2} & \text { for } x \text { in } S^{d(\mathbb{F})} \backslash\{a\} \\ \mathbb{F}(1,0) \subseteq \mathbb{F}^{2} & \text { for } x=a,\end{cases}
$$

is a biregular isomorphism. We will make use of the $\mathbb{F}$-vector bundle $\gamma_{d(\mathbb{F})}:=\alpha_{\mathbb{F}}^{*} \gamma_{2,1}(\mathbb{F})$ on $S^{d(\mathbb{F})}$.

Corollary 3.8. For any $\mathcal{C}^{r}$ map $h: X \rightarrow S^{d(\mathbb{F})}$, the following conditions are equivalent:

(a) $h$ can be approximated in the $\mathcal{C}^{r}$ topology by $\mathcal{C}^{r}$ rational maps.

(b) $h$ is homotopic to a $\mathcal{C}^{r}$ rational map.

(c) The pullback $\mathbb{F}$-vector bundle $h^{*} \gamma_{d(\mathbb{F})}$ on $X$ admits a $\mathcal{C}^{r}$ rational structure.

Proof. It suffices to apply Theorem 3.7.

There is a connection between Corollaries 2.7 and 3.8. The values of $d(\mathbb{F})$ are 1,2 or 4 , when $\mathbb{F}$ is $\mathbb{R}, \mathbb{C}$ or $\mathbb{H}$, respectively. In Corollary 2.7 , one can take $\theta_{p}=\left(\gamma_{p}\right)_{\mathbb{R}}$ for $p$ equal to one of the integers 1,2 or 4.

The example below shows that $\mathbb{F}$-vector bundles admitting a $\mathcal{C}^{r}$ rational structure are distinct from other types of $\mathbb{F}$-vector bundles studied heretofore in literature.

Example 3.9. (i) There are $\mathbb{F}$-vector bundles which do not admit a $\mathcal{C}^{r}$ rational structure for any $r$. Indeed, let $Y$ be a nonsingular real algebraic variety diffeomorphic to $S^{4} \times S^{1}$ 
and satisfying $H_{\mathrm{alg}}^{4}(Y, \mathbb{Z} / 2)=0$, cf. [18, Corollary 1.8]. Define $h: Y \rightarrow S^{4}$ by $h=\pi \circ \varphi$, where $\varphi: Y \rightarrow S^{4} \times S^{1}$ is a smooth diffeomorphism and $\pi: S^{4} \times S^{1} \rightarrow S^{4}$ is the canonical projection. The $\mathbb{H}$-vector bundle $\xi=h^{*} \gamma_{4}$ on $Y$ satisfies $w_{1}\left(\xi_{\mathbb{R}}\right) \neq 0$. By Theorem 3.6, the $\mathbb{R}$-vector bundle $\xi_{\mathbb{R}}$ does not admit a $\mathcal{C}^{r}$ rational structure. Consequently, the $\mathbb{C}$-vector bundle $\xi_{\mathbb{C}}$ and the $\mathbb{H}$-vector bundle $\xi$ do not admit a $\mathcal{C}^{r}$ rational structure. Here $\xi_{\mathbb{C}}$ denotes $\xi$ regarded as a $\mathbb{C}$-vector bundle.

(ii) Let $r$ be a nonnegative integer. There are $\mathbb{F}$-vector bundles which admit a $\mathcal{C}^{r}$ rational structure, but do not admit an algebraic structure. Indeed, by [5, Theorem 9.1], there exists a nonsingular real algebraic variety $\Sigma$ diffeomorphic to $S^{4}$ on which every $\mathbb{F}$-vector bundle admitting an algebraic structure is stably trivial. Let $\psi: \Sigma \rightarrow S^{4}$ be a smooth diffeomorphism. The $\mathbb{H}$-vector bundle $\zeta=\psi^{*} \gamma_{4}$ on $\Sigma$ satisfies $w_{4}\left(\zeta_{\mathbb{R}}\right) \neq 0$. Hence the vector bundles $\zeta_{\mathbb{R}}, \zeta_{\mathbb{C}}, \zeta$ are not stably trivial, and therefore do not admit an algebraic structure. On the other hand, by Theorem 1.2, they admit a $\mathcal{C}^{r}$ rational structure.

Assuming that $X$ is of low dimension, in the next two corollaries we give simple characterizations of $\mathbb{F}$-vector bundles on $X$ admitting a $\mathcal{C}^{r}$ rational structure.

Corollary 3.10. If $\operatorname{dim} X \leq 3$, then for any $\mathbb{R}$-vector bundle $\xi$ on $X$, the following conditions are equivalent:

(a) $\xi$ admits a $\mathcal{C}^{r}$ rational structure.

(b) $\xi$ admits an algebraic structure.

(c) $\xi$ has constant rank and $w_{i}(\xi)$ is in $H_{\mathrm{alg}}^{i}(X, \mathbb{Z} / 2)$ for $i=1,2$.

Proof. By [11, Theorem 1.6], (b) is equivalent to (c). According to Theorem 3.6, (a) implies (c). It is obvious that (b) implies (a).

Corollary 3.11. Assume that $\operatorname{dim} X \leq d(\mathbb{F})+1$ and $r$ is a nonnegative integer. For any $\mathbb{F}$-vector bundle $\xi$ on $X$, the following conditions are equivalent:

(a) $\xi$ admits a $\mathcal{C}^{r}$ rational structure.

(b) $\xi$ has constant rank and $w_{d(\mathbb{F})}\left(\xi_{\mathbb{R}}\right)$ is in $H_{\mathrm{alg}}^{d(\mathbb{F})}(X, \mathbb{Z} / 2)$.

Proof. By Theorem 3.6, (a) implies (b) (recall that every $\mathbb{F}$-vector bundle admitting a $\mathcal{C}^{r}$ rational structure has constant rank).

If $\mathbb{F}=\mathbb{R}$, then (b) implies (a) in virtue of Corollary 3.10. Assume now that (b) holds and $\mathbb{F}$ is either $\mathbb{C}$ or $\mathbb{H}$. If $\operatorname{rank} \xi=p \geq 1$, then $\xi$ is isomorphic to an $\mathbb{F}$ vector bundle of the form $\left(h^{*} \gamma_{d(\mathbb{F})}\right) \oplus \varepsilon_{X}^{p-1}(\mathbb{F})$ for some continuous map $h: X \rightarrow$ $S^{d(\mathbb{F})}$. Since $w_{d(\mathbb{F})}\left(\xi_{\mathbb{R}}\right)=h^{*}\left(w_{d(\mathbb{F})}\left(\left(\gamma_{d(\mathbb{F})}\right)_{\mathbb{R}}\right)\right)$ and $w_{d(\mathbb{F})}\left(\left(\gamma_{d(\mathbb{F})}\right)_{\mathbb{R}}\right)$ is a generator of $H_{\text {alg }}^{d(\mathbb{F})}\left(S^{d(\mathbb{F})}, \mathbb{Z} / 2\right) \cong \mathbb{Z} / 2$, by Theorem 1.5 , there exists a $\mathcal{C}^{r}$ rational map $f: X \rightarrow S^{d(\mathbb{F})}$ homotopic to $h$. Hence $\xi$ is isomorphic to $\left(f^{*} \gamma_{d(\mathbb{F})}\right) \oplus \varepsilon_{X}^{p-1}(X)$, which implies that $\xi$ admits a $\mathcal{C}^{r}$ rational structure and (a) holds.

\section{References}

[1] S. Akbulut, H. King, Polynomial equations of immersed surfaces. Pacific J. Math. 131 (1988), 209-217. MR922214 (89c:57028) Zbl 0686.14030 
[2] S. Akbulut, H. King, On approximating submanifolds by algebraic sets and a solution to the Nash conjecture. Invent. Math. 107 (1992), 87-98. MR1135465 (93d:57051) Zbl 0781.14038

[3] S. Akbulut, H. King, Topology of real algebraic sets, volume 25 of Mathematical Sciences Research Institute Publications. Springer 1992. MR1225577 (94m:57001) Zbl 0808.14045

[4] R. Benedetti, A. Tognoli, Remarks and counterexamples in the theory of real algebraic vector bundles and cycles. In: Real algebraic geometry and quadratic forms (Rennes, 1981), volume 959 of Lecture Notes in Math., 198-211, Springer 1982. MR683134 (85a:14018) Zbl 0498.14015

[5] J. Bochnak, M. Buchner, W. Kucharz, Vector bundles over real algebraic varieties. $K$-Theory 3 (1989), 271-298. MR1040403 (91b:14075) Zbl 0761.14020

[6] J. Bochnak, M. Coste, M.-F. Roy, Real algebraic geometry. Springer 1998. MR1659509 (2000a:14067) Zbl 0912.14023

[7] J. Bochnak, W. Kucharz, Algebraic approximation of mappings into spheres. Michigan Math. J. 34 (1987), 119-125. MR873026 (88h:58018) Zbl 0631.14019

[8] J. Bochnak, W. Kucharz, Realization of homotopy classes by algebraic mappings. J. Reine Angew. Math. 377 (1987), 159-169. MR887406 (88g:14016) Zbl 0619.14014

[9] J. Bochnak, W. Kucharz, On real algebraic morphisms into even-dimensional spheres. Ann. of Math. (2) 128 (1988), 415-433. MR960952 (89k:57060) Zbl

[10] J. Bochnak, W. Kucharz, Algebraic models of smooth manifolds. Invent. Math. 97 (1989), 585-611. MR1005007 (91b:14076) Zbl 0687.14023

[11] J. Bochnak, W. Kucharz, $K$-theory of real algebraic surfaces and threefolds. Math. Proc. Cambridge Philos. Soc. 106 (1989), 471-480. MR1010372 (90k:14018) Zbl 0707.14006

[12] J. Bochnak, W. Kucharz, Complete intersections in differential topology and analytic geometry. Boll. Un. Mat. Ital. B (7) 10 (1996), 1019-1041. MR1430164 (98b:57045) Zbl 0904.57013

[13] J. Bochnak, W. Kucharz, On homology classes represented by real algebraic varieties. In: Singularities Symposium - Kojasiewicz 70 (Kraków, 1996; Warsaw, 1996), volume 44 of Banach Center Publ., 21-35, Polish Acad. Sci., Warsaw 1998. MR1677394 (2000b:14080) Zbl 0915.14033

[14] A. Borel, A. Haefliger, La classe d'homologie fondamentale d'un espace analytique. Bull. Soc. Math. France 89 (1961), 461-513. MR0149503 (26 \#6990) Zbl 0102.38502

[15] G. E. Bredon, Topology and geometry. Springer 1993. MR1224675 (94d:55001) Zbl 0791.55001

[16] H. Hironaka, Resolution of singularities of an algebraic variety over a field of characteristic zero. I, II. Ann. of Math. (2) 79 (1964), 109-203; ibid. (2) 79 (1964), 205-326. MR0199184 (33 \#7333) Zbl 0122.38603

[17] S.-T. Hu, Homotopy theory. Academic Press 1959. MR0106454 (21 \#5186) Zbl 0088.38803

[18] W. Kucharz, Algebraic cycles and algebraic models of smooth manifolds. J. Algebraic Geom. 11 (2002), 101-127. MR1865915 (2002j:14065) Zbl 1060.14084

[19] J. W. Milnor, Topology from the differentiable viewpoint. The University Press of Virginia, Charlottesville, Va. 1965. MR0226651 (37 \#2239) Zbl 0136.20402

[20] J. W. Milnor, J. D. Stasheff, Characteristic classes. Princeton Univ. Press 1974. MR0440554 (55 \#13428) Zbl 0298.57008

[21] D. Mumford, Algebraic geometry. I. Springer 1976. MR0453732 (56\#11992) Zbl 0356.14002 
[22] J. Peng, Z. Tang, Algebraic maps from spheres to spheres. Sci. China Ser. A 42 (1999), 11471154. MR1749862 (2000m:55015) Zbl 0993.14002

[23] R. G. Swan, Vector bundles and projective modules. Trans. Amer. Math. Soc. 105 (1962), 264-277. MR0143225 (26 \#785) Zbl 0109.41601

[24] R. G. Swan, Topological examples of projective modules. Trans. Amer. Math. Soc. 230 (1977), 201-234. MR0448350 (56 \#6657) Zbl 0443.13005

[25] R. Wood, Polynomial maps from spheres to spheres. Invent. Math. 5 (1968), 163-168. MR0227999 (37 \#3583 ) Zbl 0204.23805

Received 12 September, 2007

W. Kucharz, Max-Planck-Institut für Mathematik, Vivatsgasse 7, 53111 Bonn, Germany and Department of Mathematics and Statistics, University of New Mexico, Albuquerque, New Mexico 87131-1141, USA

Email: kucharz@math.unm.edu 